ORLICZ CENTENARY VOLUME

BANACH CENTER PUBLICATIONS, VOLUME 64

INSTITUTE OF MATHEMATICS

POLISH ACADEMY OF SCIENCES

WARSZAWA 2004

\title{
A NOTE ON WAVELET BASES IN FUNCTION SPACES
}

\author{
HANS TRIEBEL
}

Mathematisches Institut, Friedrich-Schiller-Universität Jena, D-07740 Jena, Germany

E-mail: triebel@minet.uni-jena.de

1. Introduction and theorem. Let $n \in \mathbb{N}$ and $L_{j}=L=2^{n}-1$ if $j \in \mathbb{N}$ and $L_{0}=1$.

For any $r \in \mathbb{N}$ there are real compactly supported functions

$$
\psi_{0}(x) \in C^{r}\left(\mathbb{R}^{n}\right) \quad \text { and } \quad \psi^{l}(x) \in C^{r}\left(\mathbb{R}^{n}\right) ; \quad l=1, \ldots, L,
$$

with

$$
\int_{\mathbb{R}^{n}} x^{\alpha} \psi^{l}(x) d x=0, \quad \alpha \in \mathbb{N}_{0}^{n}, \quad|\alpha| \leq r
$$

such that

$$
\left\{2^{j \frac{n}{2}} \psi_{j m}^{l}(x): j \in \mathbb{N}_{0}, 1 \leq l \leq L_{j}, m \in \mathbb{Z}^{n}\right\}
$$

with

$$
\psi_{j m}^{l}(x)= \begin{cases}\psi_{0}(x-m) & \text { if } j=0, m \in \mathbb{Z}^{n}, l=1, \\ \psi^{l}\left(2^{j-1} x-m\right) & \text { if } j \in \mathbb{N}, m \in \mathbb{Z}^{n}, 1 \leq l \leq L,\end{cases}
$$

is an orthonormal basis in $L_{2}\left(\mathbb{R}^{n}\right)$.

The best known example of such a system of functions is the (inhomogeneous) Daubechies wavelet basis. We refer for details to [19], 3.8, pp. 96/97, formula (8.2), for the one-dimensional case, including that the father wavelet $\psi_{0}$ and the mother wavelets $\psi^{l}$ are real, and 3.9, pp. 107/108, formula (9.1), for its $n$-dimensional extension. The original version goes back to I. Daubechies, [6] and [7], Chapter 6. We refer also to [42], Chapter 4, and [16], Chapter 2. It is well known that this system remains an unconditional Schauder basis in $L_{p}\left(\mathbb{R}^{n}\right)$ with $1<p<\infty$, more generally, in the (inhomogeneous fractional) Sobolev spaces

$$
H_{p}^{s}\left(\mathbb{R}^{n}\right) \quad \text { with } \quad 1<p<\infty, \quad|s|<r,
$$

and in the (inhomogeneous) Besov spaces

$$
B_{p q}^{s}\left(\mathbb{R}^{n}\right) \quad \text { with } \quad 1 \leq p<\infty, \quad 1 \leq q<\infty, \quad|s|<r .
$$

2000 Mathematics Subject Classification: 46E35, 42B35, 42C40.

The paper is in final form and no version of it will be published elsewhere. 
Details may be found in [19], Chapter 6 , but also in the other books and papers mentioned above. It is the aim of this note to extend this assertion directly from $L_{2}\left(\mathbb{R}^{n}\right)$ to all spaces $B_{p q}^{s}\left(\mathbb{R}^{n}\right)$ and $F_{p q}^{s}\left(\mathbb{R}^{n}\right)$ with

$$
s \in \mathbb{R}, \quad 0<p \leq \infty, \quad 0<q \leq \infty,
$$

$(p<\infty$ in the $F$-case) in a comparatively simple way using only a few qualitative assertions of the recent theory of function spaces, such as

- atomic decompositions,

- characterisations by local means,

- duality.

For this purpose we need for $s, p, q$ given by (1.7) the sequence spaces $b_{p q}^{s}$ and $f_{p q}^{s}$, consisting of all sequences

$$
\lambda=\left\{\lambda_{j m}^{l} \in \mathbb{C}: j \in \mathbb{N}_{0} ; 1 \leq l \leq L_{j} ; m \in \mathbb{Z}^{n}\right\}
$$

such that the respective quasi-norms

$$
\left\|\lambda \mid b_{p q}^{s}\right\|=\left(\sum_{l, j} 2^{j\left(s-\frac{n}{p}\right) q}\left(\sum_{m}\left|\lambda_{j m}^{l}\right|^{p}\right)^{\frac{q}{p}}\right)^{\frac{1}{q}}
$$

and

$$
\left\|\lambda\left|f_{p q}^{s}\|=\|\left(\sum_{l, j, m} 2^{j s q}\left|\lambda_{j m}^{l} \chi_{j m}(\cdot)\right|^{q}\right)^{\frac{1}{q}}\right| L_{p}\left(\mathbb{R}^{n}\right)\right\|,
$$

(with the usual modifications if $p=\infty$ and/or $q=\infty$ ) are finite. Sequence spaces of this type were introduced in [11], [12] in connection with atomic decompositions of the spaces $B_{p q}^{s}\left(\mathbb{R}^{n}\right)$ and $F_{p q}^{s}\left(\mathbb{R}^{n}\right)$ and have been used afterwards by many authors. Here $\chi_{j m}$ is the characteristic function of the cube $Q_{j m}$ in $\mathbb{R}^{n}$ with sides parallel to the axes centred at $2^{-j} m$ and with side-length $2^{-j}$. As usual nowadays, $A_{p q}^{s}\left(\mathbb{R}^{n}\right)$ stands for $B_{p q}^{s}\left(\mathbb{R}^{n}\right)$ or $F_{p q}^{s}\left(\mathbb{R}^{n}\right)$, and $a_{p q}^{s}$ stands for $b_{p q}^{s}$ or $f_{p q}^{s}$, respectively.

TheOrem. Let $s, p, q$ be given by (1.7) with $p<\infty$ in the F-case. There are natural numbers $r(s, p)$ for the B-spaces and $r(s, p, q)$ for the $F$-spaces with the following properties.

(i) Let $r \in \mathbb{N}$ with $r>r(s, p)$ or $r>r(s, p, q)$, respectively. Let $f \in S^{\prime}\left(\mathbb{R}^{n}\right)$. Then $f \in A_{p q}^{s}\left(\mathbb{R}^{n}\right)$ if, and only if, it can be represented as

$$
f=\sum_{l, j, m} \lambda_{j m}^{l} \psi_{j m}^{l} \quad \text { with } \quad\left\|\lambda \mid a_{p q}^{s}\right\|<\infty,
$$

with unconditional convergence in $S^{\prime}\left(\mathbb{R}^{n}\right)$ and locally in any $A_{p u}^{\sigma}\left(\mathbb{R}^{n}\right)$ with $\sigma<s$. Furthermore, the representation (1.10) is unique,

$$
\lambda_{j m}^{l}=2^{j n}\left(f, \psi_{j m}^{l}\right)
$$

and

$$
\left\|f\left|A_{p q}^{s}\left(\mathbb{R}^{n}\right)\|\sim\| \lambda\right| a_{p q}^{s}\right\|
$$

(equivalent quasi-norms). 
(ii) In addition, let $p<\infty$ and $q<\infty$. Then (1.10) with (1.11) converges unconditionally in $A_{p q}^{s}\left(\mathbb{R}^{n}\right)$ and $\left\{\psi_{j m}^{l}\right\}$ is an unconditional Schauder basis in $A_{p q}^{s}\left(\mathbb{R}^{n}\right)$.

REMARK 1. As already mentioned, it is our aim to extend the $L_{2}\left(\mathbb{R}^{n}\right)$-theory to $A_{p q}^{s}\left(\mathbb{R}^{n}\right)$ using only a few qualitative assertions. This makes it clear that these arguments can also applied to wider classes of function spaces and to other $L_{2}\left(\mathbb{R}^{n}\right)$-bases, provided that the ingredients needed are available. This will not be done here (but we add some comments about this possibility in Subsection 3.4). By (1.5), combined with the Littlewood-Paley assertion

$$
H_{p}^{s}\left(\mathbb{R}^{n}\right)=F_{p, 2}^{s}\left(\mathbb{R}^{n}\right), \quad s \in \mathbb{R}, \quad 1<p<\infty,
$$

and (1.6) a few cases are known. Furthermore, there are also some extensions to more general spaces $B_{p q}^{s}\left(\mathbb{R}^{n}\right)$ and $F_{p q}^{s}\left(\mathbb{R}^{n}\right)$ in the literature. Nearest to us is [13], Section 7 , and [17] (Lemarié-Meyer wavelets in homogeneous $B$-spaces and $F$-spaces, and perturbations of the related bases, respectively). We refer in this context also to the respective considerations in [23] and [16].

The plan of this note is the following. In Section 2 we give some definitions (about function spaces) and we prove the Theorem. In Section 3 we discuss several consequences and prove some corollaries. In particular we estimate in Corollary 5 the above numbers $r(s, p)$ and $r(s, p, q)$ and say something about the structure of the spaces $A_{p q}^{s}\left(\mathbb{R}^{n}\right)$ in Corollary 7. It is well known that it may happen that the best possible values of $s$ in $f \in A_{p q}^{s}\left(\mathbb{R}^{n}\right)$ are getting larger (even tending to infinity) if $p \rightarrow 0$. As a consequence of the above Theorem we show in Corollary 9 that this is not the case for full spaces $A_{p q}^{s}\left(\mathbb{R}^{n}\right)$. Finally in Subsection 3.4 we add a few comments on how the techniques presented here can possibly be used in other cases.

\section{Definitions, proof of the theorem}

2.1. Basic notation. We use standard notation. Let $\mathbb{N}$ be the collection of all natural numbers and let $\mathbb{N}_{0}=\mathbb{N} \cup\{0\}$. Let $\mathbb{R}^{n}$ be euclidean $n$-space, where $n \in \mathbb{N}$; put $\mathbb{R}=\mathbb{R}^{1}$, whereas $\mathbb{C}$ is the complex plane. Let $S\left(\mathbb{R}^{n}\right)$ be the Schwartz space of all complex-valued rapidly decreasing, infinitely differentiable functions on $\mathbb{R}^{n}$. By $S^{\prime}\left(\mathbb{R}^{n}\right)$ we denote its topological dual, the space of tempered distributions on $\mathbb{R}^{n}$. Furthermore, $L_{p}\left(\mathbb{R}^{n}\right)$ with $0<p \leq \infty$, is the standard quasi-Banach space with respect to Lebesgue measure, quasi-normed by

$$
\left\|f \mid L_{p}\left(\mathbb{R}^{n}\right)\right\|=\left(\int_{\mathbb{R}^{n}}|f(x)|^{p} d x\right)^{\frac{1}{p}}
$$

with the obvious modification if $p=\infty$. Let $C\left(\mathbb{R}^{n}\right)$ be the Banach space of all complexvalued uniformly continuous bounded functions in $\mathbb{R}^{n}$ and let for $r \in \mathbb{N}$,

$$
C^{r}\left(\mathbb{R}^{n}\right)=\left\{f \in C\left(\mathbb{R}^{n}\right): D^{\alpha} f \in C\left(\mathbb{R}^{n}\right),|\alpha| \leq r\right\},
$$

obviously normed.

As usual, $\mathbb{Z}$ is the collection of all integers; and $\mathbb{Z}^{n}$ where $n \in \mathbb{N}$, denotes the lattice of all points $m=\left(m_{1}, \ldots, m_{n}\right) \in \mathbb{R}^{n}$ with $m_{j} \in \mathbb{Z}$. Let $\mathbb{N}_{0}^{n}$, where $n \in \mathbb{N}$, be the set of 
all multi-indices

$$
\alpha=\left(\alpha_{1}, \ldots, \alpha_{n}\right) \quad \text { with } \quad \alpha_{j} \in \mathbb{N}_{0} \quad \text { and } \quad|\alpha|=\sum_{j=1}^{n} \alpha_{j} .
$$

If $x=\left(x_{1}, \ldots, x_{n}\right) \in \mathbb{R}^{n}$ and $\alpha=\left(\alpha_{1}, \ldots, \alpha_{n}\right) \in \mathbb{N}_{0}^{n}$ then we put

$$
x^{\alpha}=x_{1}^{\alpha_{1}} \cdots x_{n}^{\alpha_{n}} \quad \text { (monomials). }
$$

Now all notation used in Section 1 is defined with exception of the spaces $B_{p q}^{s}\left(\mathbb{R}^{n}\right)$ and $F_{p q}^{s}\left(\mathbb{R}^{n}\right)$.

2.2. Function spaces. If $\varphi \in S\left(\mathbb{R}^{n}\right)$ then

$$
\widehat{\varphi}(\xi)=(F \varphi)(\xi)=(2 \pi)^{-\frac{n}{2}} \int_{\mathbb{R}^{n}} e^{-i \xi x} \varphi(x) d x, \quad \xi \in \mathbb{R}^{n},
$$

denotes the Fourier transform of $\varphi$. Here $\xi x$ is the scalar product in $\mathbb{R}^{n}$. As usual, $F^{-1} \varphi$ or $\varphi^{\vee}$, stands for the inverse Fourier transform, given by the right-hand side of (2.1) with $i$ in place of $-i$. Both $F$ and $F^{-1}$ are extended to $S^{\prime}\left(\mathbb{R}^{n}\right)$ in the standard way.

Let $\varphi \in S\left(\mathbb{R}^{n}\right)$ with

$$
\varphi(x)=1 \text { if }|x| \leq 1 \text { and } \quad \varphi(y)=0 \text { if }|y| \geq \frac{3}{2} .
$$

We put $\varphi_{0}=\varphi$. Let $\varphi_{1}(x)=\varphi\left(\frac{x}{2}\right)-\varphi(x)$ and

$$
\varphi_{k}(x)=\varphi_{1}\left(2^{-k+1} x\right), \quad x \in \mathbb{R}^{n}, \quad k \in \mathbb{N} .
$$

Then $\sum_{k=0}^{\infty} \varphi_{k}(x)=1$ for all $x \in \mathbb{R}^{n}$ is a dyadic resolution of unity. Recall that $\left(\varphi_{k} \widehat{f}\right)^{\vee}$ is an entire analytic function and hence $\left(\varphi_{k} \widehat{f}\right)^{\vee}(x)$ makes sense pointwise.

DeFinition 2. Let $\varphi$ and $\varphi_{j}$ be the above functions.

(i) Let $s \in \mathbb{R}, 0<p \leq \infty$, and $0<q \leq \infty$. Then $B_{p q}^{s}\left(\mathbb{R}^{n}\right)$ is the collection of all $f \in S^{\prime}\left(\mathbb{R}^{n}\right)$ such that

$$
\left\|f \mid B_{p q}^{s}\left(\mathbb{R}^{n}\right)\right\|_{\varphi}=\left(\sum_{j=0}^{\infty} 2^{j s q}\left\|\left(\varphi_{j} \widehat{f}\right)^{\vee} \mid L_{p}\left(\mathbb{R}^{n}\right)\right\|^{q}\right)^{\frac{1}{q}}
$$

(with the usual modification if $q=\infty$ ) is finite.

(ii) Let $s \in \mathbb{R}, 0<p<\infty$, and $0<q \leq \infty$. Then $F_{p q}^{s}\left(\mathbb{R}^{n}\right)$ is the collection of all $f \in S^{\prime}\left(\mathbb{R}^{n}\right)$ such that

$$
\left\|f\left|F_{p q}^{s}\left(\mathbb{R}^{n}\right)\left\|_{\varphi}=\right\|\left(\sum_{j=0}^{\infty} 2^{j s q}\left|\left(\varphi_{j} \widehat{f}\right)^{\vee}(\cdot)\right|^{q}\right)^{\frac{1}{q}}\right| L_{p}\left(\mathbb{R}^{n}\right)\right\|
$$

(with the usual modification if $q=\infty$ ) is finite.

REMARK 3. The theory of these spaces in its full extent has been developed in [36] and [37]. But they have a long history, including their forerunners and special cases. A few of them will be mentioned in the next remark. The interested reader may consult Chapter 1 in [37] which is a historically-minded survey from the beginnings up to the early nineties. The theory of these spaces as it stood in the middle of the nineties may be found in [1], [8] and [23]. As for some more recent aspects we refer to [38] and [39]. 
REMARK 4. The spaces $B_{p q}^{s}\left(\mathbb{R}^{n}\right)$ and $F_{p q}^{s}\left(\mathbb{R}^{n}\right)$ are quasi-Banach spaces. They are independent of $\varphi$ : different choices of admissible functions $\varphi$ result in (2.4) and (2.5) in equivalent quasi-norms, respectively. This may justify our omission of the subscript $\varphi$ on the left-hand sides of (2.4) and (2.5) in the sequel. We list a few special cases. Recall that for any $\sigma \in \mathbb{R}$,

$$
I_{\sigma}: \quad f \mapsto\left(\left(1+|\xi|^{2}\right)^{\frac{\sigma}{2}} \widehat{f}\right)^{\vee}
$$

is an one-to-one map of $S\left(\mathbb{R}^{n}\right)$ onto itself and of $S^{\prime}\left(\mathbb{R}^{n}\right)$ onto itself. Then

$$
H_{p}^{s}\left(\mathbb{R}^{n}\right)=I_{-s} L_{p}\left(\mathbb{R}^{n}\right), \quad s \in \mathbb{R}, \quad 1<p<\infty,
$$

are the (fractional) Sobolev spaces with the classical Sobolev spaces

$$
W_{p}^{k}\left(\mathbb{R}^{n}\right)=H_{p}^{k}\left(\mathbb{R}^{n}\right), \quad k \in \mathbb{N}_{0}, \quad 1<p<\infty,
$$

as a subclass, where the latter spaces can be equivalently normed by

$$
\left\|f\left|W_{p}^{k}\left(\mathbb{R}^{n}\right)\left\|=\sum_{|\alpha| \leq k}\right\| D^{\alpha} f\right| L_{p}\left(\mathbb{R}^{n}\right)\right\| .
$$

For these spaces one has the above-mentioned Littlewood-Paley characterisation

$$
H_{p}^{s}\left(\mathbb{R}^{n}\right)=F_{p, 2}^{s}\left(\mathbb{R}^{n}\right), \quad s \in \mathbb{R}, \quad 1<p<\infty .
$$

Furthermore,

$$
B_{p q}^{s}\left(\mathbb{R}^{n}\right), \quad s>0, \quad 1<p<\infty, \quad 1 \leq q \leq \infty,
$$

are the classical Besov spaces. Let

$$
\mathcal{C}^{s}\left(\mathbb{R}^{n}\right)=B_{\infty \infty}^{s}\left(\mathbb{R}^{n}\right), \quad s \in \mathbb{R} .
$$

Then $\mathcal{C}^{s}\left(\mathbb{R}^{n}\right)$ with $s>0$ are the Hölder-Zygmund spaces. If $0<p \leq 1$ then $h_{p}\left(\mathbb{R}^{n}\right)=$ $F_{p, 2}^{0}\left(\mathbb{R}^{n}\right)$ are the (inhomogeneous) Hardy spaces.

2.3. Duality. The basic ideas of the proof are rather simple. But there are a few technicalities which must be treated with some care and which originate mainly from the limited smoothness of the functions $\psi_{j m}^{l}$. In particular one has to clarify the meaning of the right-hand side of (1.11) if

$$
f \in A_{p q}^{s}\left(\mathbb{R}^{n}\right), \quad s, p, q \text { as in }(1.7),
$$

(always with $p<\infty$ for the $F$-spaces). This is a matter of the duality theory as developed in [36], 2.11. Let

$$
\sigma_{p}=n\left(\frac{1}{p}-1\right)_{+}, \quad 0<p \leq \infty,
$$

where $a_{+}=\max (0, a)$ if $a \in \mathbb{R}$. Let $\frac{1}{p}+\frac{1}{p^{\prime}}=1$ if $1 \leq p \leq \infty$ and $p^{\prime}=\infty$ if $0<p<1$. According to [36], Theorems 2.11.2 and 2.11.3, one has for the dual spaces of $B_{p p}^{s}\left(\mathbb{R}^{n}\right)$,

$$
B_{p p}^{s}\left(\mathbb{R}^{n}\right)^{\prime}=B_{p^{\prime} p^{\prime}}^{-s+\sigma_{p}}\left(\mathbb{R}^{n}\right), \quad s \in \mathbb{R}, \quad 0<p<\infty .
$$

This assertion can be extended to $p=\infty$ if one replaces $\mathcal{C}^{s}\left(\mathbb{R}^{n}\right)=B_{\infty \infty}^{s}\left(\mathbb{R}^{n}\right)$ on the left-hand side of $(2.9)$ by $\mathcal{C}^{s}\left(\mathbb{R}^{n}\right)$, the completion of $S\left(\mathbb{R}^{n}\right)$ in $\mathcal{C}^{s}\left(\mathbb{R}^{n}\right)$. By elementary 
embeddings, (2.9), and the compactness of the supports of $\psi_{j m}^{l}$ it follows that the righthand side of (1.11) is well defined if

$$
r>-s+\sigma_{p}, \quad s \in \mathbb{R}, \quad 0<p \leq \infty .
$$

Hence for fixed $s$, minimal numbers $r(s, p)$ and $r(s, p, q)$ in the theorem have to tend to infinity as $\frac{n}{p}$ if $p \rightarrow 0$.

2.4. Proof of the theorem

Step 1. Let $f \in S^{\prime}\left(\mathbb{R}^{n}\right)$ be given by (1.10) and let

$$
r>\max \left(s, \sigma_{p}-s-1\right)
$$

in the $B$-case and

$$
r>\max \left(s, \sigma_{p q}-s-1\right)
$$

in the $F$-case, where $\sigma_{p}$ is given by (2.8) and

$$
\sigma_{p q}=n\left(\frac{1}{\min (p, q)}-1\right)_{+}, \quad 0<p<\infty, \quad 0<q \leq \infty .
$$

Then $2^{-j\left(s-\frac{n}{p}\right)} \psi_{j m}^{l}$ are normalised atoms in $A_{p q}^{s}\left(\mathbb{R}^{n}\right)$. We refer to [38], Theorem 13.8, pp. $75 / 76$. But as mentioned above the theory of atomic decompositions in $A_{p q}^{s}\left(\mathbb{R}^{n}\right)$ goes back to [11], [12]. We refer also to [13], [33]. Hence, $f \in A_{p q}^{s}\left(\mathbb{R}^{n}\right)$ and

$$
\left\|f\left|A_{p q}^{s}\left(\mathbb{R}^{n}\right)\|\leq c\| \lambda\right| a_{p q}^{s}\right\|
$$

where $c$ is independent of $\lambda \in a_{p q}^{s}$.

Step 2. Conversely let $f \in A_{p q}^{s}\left(\mathbb{R}^{n}\right)$ and let $\lambda_{j m}^{l}$ be given by (1.11). We start with some preparations. If $(2.11),(2.12)$ are slightly strengthened by $(2.10)$ then at least (1.11) makes sense within the dual pairing $\left(S\left(\mathbb{R}^{n}\right), S^{\prime}\left(\mathbb{R}^{n}\right)\right)$. But we claim that (1.11) are even local means in the $F$-spaces and in the $B$-spaces according to [37], 2.4.6, p. 122, and 2.5.3, p. 138, respectively, extended to non- $C^{\infty}$ kernels if $r$ is chosen sufficiently large. All what one needs is covered by [37], 2.4 ( $F$-spaces) and 2.5 ( $B$-spaces). But we give a detailed description indicating the needed ingredients. We deal with the $F$-case (the $B$-case is similar, but technically simpler). Let $r \in \mathbb{N}$ and

$$
\psi \in C^{r}\left(\mathbb{R}^{n}\right) \text { real with } \operatorname{supp} \psi \text { compact. }
$$

Then for $t>0$ and with the usual interpretation,

$$
\psi(t, f)(x)=\int_{\mathbb{R}^{n}} \psi(y) f(x+t y) d y=t^{-n} \int_{\mathbb{R}^{n}} \psi\left(\frac{y-x}{t}\right) f(y) d y
$$

are the local means. In particular, with $\psi=\psi^{l}$ according to (1.1), $x=2^{-j} m$ and $t=2^{-j}$ one gets

$$
\begin{aligned}
\psi^{l}\left(2^{-j}, f\right)\left(2^{-j} m\right) & =2^{j n} \int_{\mathbb{R}^{n}} \psi^{l}\left(2^{j} y-m\right) f(y) d y=2^{j n} \int_{\mathbb{R}^{n}} \psi_{j m}^{l}(y) f(y) d y \\
& =\lambda_{j m}^{l}(f),
\end{aligned}
$$

where we used (1.4) and (1.11), now indicating the (linear) dependence of $\lambda_{j m}^{l}$ on $f$. Similarly for $\psi_{0}$ in (1.1) in place of $\psi^{l}$. Because of the lack of the $C^{\infty}$-smoothness of $\psi_{0}$ and $\psi^{l}$ in (1.1) and also because we wish to end up with (1.12) we must say how 
the technicalities in [37], 2.4, have to be adapted. We rely on a modified version of the equivalent quasi-norm in $F_{p q}^{s}\left(\mathbb{R}^{n}\right)$ according to [37], Corollary 2, pp. 108/109, in terms of maximal functions. We put $\psi=\psi^{l}$ according to (1.1) and identify the function $\varphi$ in [37], p. 109, formula (55), with the entire analytic function $\psi^{\vee}$. Then an easy calculation gives

$$
\psi_{j}^{\vee}(D) f(x-y)=2^{j n} \int_{\mathbb{R}^{n}} \psi\left(2^{j} z+2^{j} y-2^{j} x\right) f(z) d z=\psi\left(2^{-j}, f\right)(x-y),
$$

where $x \in \mathbb{R}^{n}, y \in \mathbb{R}^{n}$ and $j \in \mathbb{N}$, connecting the notation used in [37] and in (2.15). The modified maximal function in [37], p. 109, formula (55), is given by

$$
\psi\left(2^{-j}, f\right)^{+}(x)=\sup _{|y| \leq \sqrt{n} 2^{-j}}\left|\psi\left(2^{-j}, f\right)(x-y)\right|, \quad x \in \mathbb{R}^{n} .
$$

One must check whether the hypotheses of [37], Corollary 2, pp. 108/109, are satisfied. As for the Tauberian condition we refer to [37], 2.4.4, p. 120, Proposition 2, making it clear (after a dilation) that it is sufficient to know that the entire analytic functions $\psi_{0}^{\vee}$ on the one hand, and $\left(\psi^{l}\right)^{\vee}, l=1, \ldots, L$, together on the other hand, satisfy this condition for some $\varepsilon>0$ in a ball $\{x:|x| \leq 2 \varepsilon\}$ as far as $\psi_{0}^{\vee}$ is concerned and in an annulus $\{x: \varepsilon \leq|x| \leq 4 \varepsilon\}$ as far as the $\left(\psi^{l}\right)^{\vee}$ 's are concerned. But this is the case. Concerning the cancellation conditions, expressed by formula (50) and $s_{1}>\sigma_{p}$ in formula (54) on p. 108 in [37] we refer to the improved version in [24] saying that $r>s-1$ is sufficient. But this is covered by (2.12). It remains to check the following condition. Let $H \in S\left(\mathbb{R}^{n}\right)$ with

$$
H(x)=1 \text { if } \frac{1}{2} \leq|x| \leq 2 \text { and } H(y)=0 \text { if }|y| \leq \frac{1}{4} \text { or }|y| \geq 4
$$

and let $s_{0} \in \mathbb{R}$ be a number with

$$
s_{0}+\sigma_{p q}<s
$$

where $\sigma_{p q}$ is given by $(2.13)$. Let

$$
\sup _{k \in \mathbb{N}} 2^{-k s_{0}}\left\|\psi^{\vee}\left(2^{k} \cdot\right) H(\cdot) \mid H_{2}^{\sigma}\left(\mathbb{R}^{n}\right)\right\|<\infty,
$$

where $H_{2}^{\sigma}\left(\mathbb{R}^{n}\right)$ is the special Sobolev space according to (2.6) with $\sigma>\frac{n}{2}+\frac{n}{\min (p, q)}$. Then we claim that, in obvious notation, adapted to (1.9),

$$
\left\|f\left|F_{p q}^{s}\left(\mathbb{R}^{n}\right)\|\sim\|\left(\sum_{l, j, m} 2^{j s q} \psi_{j}^{l,+}(\cdot)^{q}\right)^{\frac{1}{q}}\right| L_{p}\left(\mathbb{R}^{n}\right)\right\|
$$

where $\psi_{j}^{l,+}$ are the maximal functions according to $(2.17)$ with $\psi^{l}$ and $\psi_{0}$ in place of $\psi$. The estimates of the left-hand side of (2.20) from below and from above by the right-hand side follow from the two corollaries in [37], pp. 108/109. But it is quite clear that (2.19) with (2.18) is satisfied if $r$ in (1.1) is large. In Corollary 5 we return to this point and give an explicit estimate for $r$.

Step 3. Again let $f \in A_{p q}^{s}\left(\mathbb{R}^{n}\right)$ and as in the previous step we assume $A=F$ (the case $A=B$ is similar and simpler). By (2.20) with (2.17), and (1.9) with (2.16) we have

$$
\left\|\lambda(f)\left|f_{p q}^{s}\|\leq c\| f\right| F_{p q}^{s}\left(\mathbb{R}^{n}\right)\right\|<\infty .
$$


Then by Step 1

$$
g=\sum_{l, j, m} \lambda_{j m}^{l}(f) \psi_{j m}^{l} \in F_{p q}^{s}\left(\mathbb{R}^{n}\right)
$$

Using that (1.3) is an orthonormal system and the duality according to Subsection 2.3 it follows

$$
\left(g, \psi_{j^{\prime} m^{\prime}}^{l^{\prime}}\right)=\sum_{l, j, m} 2^{j n}\left(f, \psi_{j m}^{l}\right)\left(\psi_{j m}^{l}, \psi_{j^{\prime} m^{\prime}}^{l^{\prime}}\right)=\left(f, \psi_{j^{\prime} m^{\prime}}^{l^{\prime}}\right) .
$$

This can be extended to finite linear combinations of $\psi_{j^{\prime} m^{\prime}}^{l^{\prime}}$. If $\varphi \in S\left(\mathbb{R}^{n}\right)$ then we have the unique $L_{2}\left(\mathbb{R}^{n}\right)$-representation. By the above considerations it is also a representation in the spaces described in (2.9) with (2.10). Now it follows from (2.23) that

$$
(g, \varphi)=(f, \varphi) \quad \text { for all } \varphi \in S\left(\mathbb{R}^{n}\right)
$$

and hence $g=f$.

Step 4. So far we proved that $f$ belongs to $A_{p q}^{s}\left(\mathbb{R}^{n}\right)$ if, and only if, it can be represented by (1.10) and that this representation is unique with the coefficients in (1.11), now denoted by $\lambda_{j m}^{l}(f)$. Again let $A=F$. We have (2.21). The converse follows from (2.14) and (2.22) with $g=f$. This proves (1.12). The unconditional convergence of $f$ given by (1.10) in $A_{p u}^{\sigma}\left(\mathbb{R}^{n}\right)$ with $\sigma<s$, and hence in $S^{\prime}\left(\mathbb{R}^{n}\right)$, follows from the structure of the sequence spaces in (1.8), (1.9) and $\sigma<s$. This proves part (i) of the theorem. If $p<\infty$ and $q<\infty$ then one has even unconditional convergence in $A_{p q}^{s}\left(\mathbb{R}^{n}\right)$. This proves part (ii) of the theorem.

\section{Complements}

3.1. Smoothness of wavelets. At several occasions during the proof of the theorem we used that $r \in \mathbb{N}$ in (1.1) must be sufficiently large. The restrictions in (1.5) and (1.6) are optimal. But something like this cannot be expected in general. By (2.10) with (2.8) one has necessarily that

$$
r(s, p, q) \geq r(s, p) \geq-s+\sigma_{p} .
$$

The first inequality is reasonable. One can prove it by interpolation. In other words, if $p \rightarrow 0$ then $r(s, p)$ tends to infinity as $\frac{n}{p}$.

Corollary 5. Let s, $p, q$ be given by (1.7) with $p<\infty$ in the F-case. Then one can choose in the Theorem

$$
r(s, p)=\max \left(s, \frac{2 n}{p}+\frac{n}{2}-s\right) \quad \text { in the } B \text {-case }
$$

and

$$
r(s, p, q)=\max \left(s, \frac{2 n}{\min (p, q)}+\frac{n}{2}-s\right) \quad \text { in the } F \text {-case. }
$$

Proof. Step 1. First we remark that these numbers cover (2.10) and (2.11), (2.12), hence duality and atomic representations. In other words, by Step 2 of the proof of the theorem we have to show that (2.19) with

$$
s_{0}+\sigma_{p q}<s \quad \text { and } \quad \sigma>\frac{n}{2}+\frac{n}{\min (p, q)} \quad \text { in the } F \text {-case }
$$


and

$$
s_{0}+\sigma_{p}<s \text { and } \sigma>\frac{n}{2}+\frac{n}{p} \text { in the } B \text {-case }
$$

is satisfied. These requirements for the $F$-spaces are covered by Step 2 of the above proof of the theorem. The counterparts for the simpler $B$-spaces are given by the two corollaries in [37], p. 134.

Step 2. We prove that (3.2) is sufficient in the $F$-case. The proof for the $B$-case resulting in (3.1) is the same. Let $\psi$ be either $\psi_{0}$ or $\psi^{l}$ according to (1.1). Recall that $\psi$ has a compact support. By (2.1) we have

$$
x^{\alpha} \psi^{\vee}(x)=c \int_{\mathbb{R}^{n}} e^{i x \xi} D^{\alpha} \psi(\xi) d \xi, \quad|\alpha| \leq r
$$

a corresponding estimate for $D^{\beta} \psi^{\vee}$, and hence

$$
\left|D^{\beta} \psi^{\vee}(x)\right| \leq c 2^{-k r} \quad \text { if } \quad|x| \sim 2^{k}, \quad k \in \mathbb{N}, \quad|\beta| \leq \sigma .
$$

Inserting this estimate in (2.19) one gets

$$
2^{-k s_{0}}\left\|\psi^{\vee}\left(2^{k} \cdot\right) H(\cdot) \mid H_{2}^{\sigma}\left(\mathbb{R}^{n}\right)\right\| \leq c 2^{-k\left(s_{0}-\sigma+r\right)} .
$$

This is clear if $\sigma \in \mathbb{N}_{0}$, otherwise it follows by interpolation of the $H_{2}^{\sigma}\left(\mathbb{R}^{n}\right)$-spaces. Now (3.2) is a consequence of $r \geq \sigma-s_{0}$ and (3.3).

REMARK 6. Obviously, the estimates (3.1), (3.2) are not optimal. They are spoilt by the use of maximal functions as in (2.20). They can surely be improved by avoiding maximal functions (if possible), especially in the $B$-case, duality

$$
r(s, p)=r\left(-s, p^{\prime}\right), \quad 1 \leq p \leq \infty, \quad \frac{1}{p}+\frac{1}{p^{\prime}}=1,
$$

and interpolation. But this will not be done here.

3.2. The structure of function spaces. The sequence spaces $b_{p q}^{s}, f_{p q}^{s}$, and $a_{p q}^{s}$ have the same meaning as in Section 1. We have $b_{p p}^{s}=f_{p p}^{s} \cong \ell_{p}$ (where $\cong$ means isomorphic) for $0<p \leq \infty$. Of course, $\ell_{p}$ is the usual sequence spaces of $p$-summable sequences of complex numbers.

COROLlary 7. (i) Under the hypotheses of the Theorem,

$$
I: f \mapsto\left\{2^{j n}\left(f, \psi_{j m}^{l}\right): l, j, m \text { as there }\right\}
$$

is an isomorphic map of $A_{p q}^{s}\left(\mathbb{R}^{n}\right)$ onto $a_{p q}^{s}$.

(ii) Let $0<p \leq \infty$ and $s \in \mathbb{R}$. Then $B_{p p}^{s}\left(\mathbb{R}^{n}\right)$ is isomorphic to $\ell_{p}$.

Proof. By the Theorem $I$ is a one-to-one map of $A_{p q}^{s}\left(\mathbb{R}^{n}\right)$ onto a (sub)space of $a_{p q}^{s}$. Let $\lambda \in a_{p q}^{s}$. Then by (2.22),

$$
g=\sum_{l, j, m} \lambda_{j m}^{l} \psi_{j m}^{l} \in A_{p q}^{s}\left(\mathbb{R}^{n}\right)
$$

and as in (2.23),

$$
\left(g, \psi_{j m}^{l}\right)=2^{-j n} \lambda_{j m}^{l} .
$$

Hence the range of $I$ is $a_{p q}^{s}$. This proves (i) and also (ii). 
REMARK 8. Assertions of this type have some history. One has

$$
\mathcal{C}^{s}\left(\mathbb{R}^{n}\right)=B_{\infty \infty}^{s}\left(\mathbb{R}^{n}\right) \cong \ell_{\infty}, \quad s \in \mathbb{R},
$$

for the Hölder-Zygmund spaces according to (2.7). We refer to [4] for a corresponding assertion on intervals. Part (i) for the $B$-spaces and in particular part (ii) with $1<p<\infty$ goes back to [34], [35], 2.11.2, and [21], pp. 180/190. The corresponding assertion for $0<p \leq 1$ looks like mathematical folklore. It seems to be known since some time, but we could not find a reference. The structure of $F_{p q}^{s}\left(\mathbb{R}^{n}\right)$ is more complicated. According to [18] the homogeneous Hardy spaces $H_{1}\left(\mathbb{R}^{n}\right)$ and then also the inhomogeneous Hardy spaces $h_{1}\left(\mathbb{R}^{n}\right)=F_{1,2}^{0}\left(\mathbb{R}^{n}\right)$ are not isomorphic to each other for different values of $n \in \mathbb{N}$ (and hence it would be better to denote them by $f_{p q}^{s}\left(\mathbb{R}^{n}\right)$ instead of $f_{p q}^{s}$ ).

3.3. There is no reverse Sobolev lemma. Let

$$
-\infty<s_{1}<s_{0}<\infty, \quad 0<p_{0}<p_{1} \leq \infty, \quad s_{0}-\frac{n}{p_{0}}=s_{1}-\frac{n}{p_{1}},
$$

and $0<q \leq \infty$. Then

$$
B_{p_{0} q}^{s_{0}}\left(\mathbb{R}^{n}\right) \hookrightarrow B_{p_{1} q}^{s_{1}}\left(\mathbb{R}^{n}\right)
$$

is a sharp embedding of Sobolev type: One loses smoothness $\left(s_{1}\right.$ is smaller than $\left.s_{0}\right)$ and gains integrability $\left(p_{1}\right.$ is larger than $\left.p_{0}\right)$. It is well known that for $f \in S^{\prime}\left(\mathbb{R}^{n}\right)$, say, with compact support, there is a converse effect. Let

$$
s_{f}(t)=\sup \left\{s: f \in B_{p \infty}^{s}\left(\mathbb{R}^{n}\right)\right\} \quad \text { where } \quad 0<t=\frac{1}{p} \leq \infty .
$$

Then $s_{f}(t)$ is a non-decreasing concave curve in an $(t, s)$-diagram with slope at most $n$ which may tend to infinity if $t \rightarrow \infty$. Problems of this type attracted some attention recently in connection with continuous functions but also with (multi)fractal Radon measures. One may consult our recent contributions [41] and [40] and the references given there. But nothing like this can be expected for full spaces reversing (3.4). There are functions $f$ for which $s_{f}(t)$ is constant. Using the above theorem one can even prove a sharper assertion.

Corollary 9. Let $s \in \mathbb{R}$ and let

$$
f_{s}=\sum_{j . l, m} \lambda_{j m}^{l} \psi_{j m}^{l}
$$

with

$$
\lambda_{j m}^{l}= \begin{cases}2^{-j s} & \text { if }|m| \leq 2^{j} \\ 0 & \text { otherwise, }\end{cases}
$$

and $r>\max \left(s, \frac{n}{2}-s\right)$ according to the Theorem. Let $0<p \leq \infty$ (with $p<\infty$ in the F-case). Then

$$
f_{s} \in A_{p q}^{s}\left(\mathbb{R}^{n}\right) \quad \text { if, and only if, } q=\infty .
$$

Proof. Step 1. By Corollary 5 we can apply the Theorem if $p$, and in case the $F$-spaces also $q$, are sufficiently large. For fixed $j$ (and $l$ ) we have that

$$
2^{j\left(s-\frac{n}{p}\right)}\left(\sum_{m}\left|\lambda_{j m}^{l}\right|^{p}\right)^{\frac{1}{p}} \sim 1 .
$$


Hence for large $p$ it follows that

$$
f_{s} \in B_{p \infty}^{s}\left(\mathbb{R}^{n}\right) \text { and } f_{s} \notin B_{p q}^{s}\left(\mathbb{R}^{n}\right) \text { if } 0<q<\infty .
$$

Furthermore by (1.9) and the compactness of the support of $f_{s}$ we get for large $p$,

$$
f_{s} \in F_{p \infty}^{s}\left(\mathbb{R}^{n}\right) .
$$

For bounded domains $\Omega$ and given $s$ and $q$ one has

$$
A_{p_{0} q}^{s}(\Omega) \hookrightarrow A_{p_{1} q}^{s}(\Omega) \text { where } 0<p_{1} \leq p_{0} \leq \infty,
$$

$\left(p_{0}<\infty\right.$ in the $F$-case). This proves the if part in (3.5) for all admitted $p$.

Step 2. By (3.6) we have for large $p$ also the only-if part for the $B$-spaces. Assuming

$$
f_{s} \in B_{p_{1} q_{1}}^{s}\left(\mathbb{R}^{n}\right) \text { for some } 0<p_{1}<\infty, 0<q_{1}<\infty \text {. }
$$

We have also $f_{s} \in B_{\infty \infty}^{s}\left(\mathbb{R}^{n}\right)$. Then it follows by (2.4) and Hölder's inequality that

$$
f_{s} \in B_{p_{\theta} q_{\theta}}^{s}\left(\mathbb{R}^{n}\right) \quad \text { if } \quad 0<\theta<1 \quad \text { and } \quad p_{\theta}=\frac{p_{1}}{\theta}, \quad q_{\theta}=\frac{q_{1}}{\theta} .
$$

For small values of $\theta$ one gets a contradiction to (3.6). The remaining assertion for the $F$-spaces follows from

$$
F_{p q}^{s}\left(\mathbb{R}^{n}\right) \hookrightarrow B_{p, \max (p, q)}^{s}\left(\mathbb{R}^{n}\right) \quad \text { where } p<\infty \text { and } q<\infty .
$$

3.4. Comments. The proof of the Theorem is qualitative: It is based on the one hand on atomic decompositions and local means in the function spaces considered, and on the other hand on the fact that the functions of the chosen $L_{2}\left(\mathbb{R}^{n}\right)$-wavelet basis are simultaneously atoms and kernels of those local means. In addition duality and occasionally (maybe even avoidable) some maximal functions are used. In other words, if other $L_{2}\left(\mathbb{R}^{n}\right)$-wavelet bases than the ones considered (Daubechies wavelet bases) fulfil these qualitative requirements then the Theorem can be extended to these wavelet bases (for example to the Lemarié-Meyer wavelets). Of at least equal interest is the question to extend the Theorem to other scales of function spaces. As already mentioned, what one mainly needs are

- atomic decompositions and

- characterisations by local means,

in these function spaces. By the recent theory of function spaces there are several candidates which may fit in this scheme. We describe a few examples, restricting ourselves mainly to references.

EXAMPLE 10. Let $w(x)$ be a weight function in $\mathbb{R}^{n}$ with

$$
0<w(x) \leq c w(y)\left(1+|x-y|^{\alpha}\right), \quad x \in \mathbb{R}^{n}, \quad y \in \mathbb{R}^{n},
$$

for some $c>0$ and $\alpha \geq 0$, and

$$
\left|D^{\gamma} w(x)\right| \leq c_{\gamma} w(x), \quad x \in \mathbb{R}^{n},
$$

for $\gamma \in \mathbb{N}_{0}^{n}$ and $c_{\gamma}>0$. Then $A_{p q}^{s}\left(\mathbb{R}^{n}, w\right)$ are defined as in Definition 2 replacing

$$
\left\|g \mid L_{p}\left(\mathbb{R}^{n}\right)\right\| \quad \text { by } \quad\left\|w g \mid L_{p}\left(\mathbb{R}^{n}\right)\right\| \text {. }
$$


Then

$$
\left\|f\left|A_{p q}^{s}\left(\mathbb{R}^{n}, w\right)\|\sim\| w f\right| A_{p q}^{s}\left(\mathbb{R}^{n}\right)\right\|
$$

is a crucial observation of the theory of these spaces. We refer to [8], Chapter 4, which, in turn, is based on [14], [15]. All one needs can be carried over from $A_{p q}^{s}\left(\mathbb{R}^{n}\right)$ to $A_{p q}^{s}\left(\mathbb{R}^{n}, w\right)$. One arrives at a counterpart of the Theorem with sequence spaces obviously modified.

EXAMPLE 11. There are atomic decompositions and local means for other types of weights in $\mathbb{R}^{n}$, including $w(x) \sim e^{c|x|^{\beta}}, c \in \mathbb{R}, 0<\beta<1$, based on ultra-distributions, and $e^{c|x|}, c \in \mathbb{R}$. In the latter case even the definitions of corresponding spaces $A_{p q}^{s}\left(\mathbb{R}^{n}, w\right)$ rely on local means. One may consult [27], [28], [29] and the references given there. There is a question to find $L_{2}\left(\mathbb{R}^{n}\right)$-wavelet bases which fit in this scheme. In case of Muckenhoupt weights we refer to [22]. The combination of Muckenhoupt weights and weights which even may grow exponentially has been given in [25].

EXAMPLE 12. In recent times spaces of generalised smoothness in $\mathbb{R}^{n}$ attracted some attention. The interest comes from the spectral theory of fractal drums where it is reasonable to study spaces $A_{p q}^{(s, \Psi)}\left(\mathbb{R}^{n}\right)$ of perturbed smoothness $s$. One may think about $2^{j s}(j+1)^{b}$ with $b \in \mathbb{R}$ in $(2.4),(2.5)$ in place of $2^{j s}$. We refer to [39], Section 22. A detailed account of these spaces has been given in [20], including atoms and local means. One may extend these considerations replacing $2^{j s}$ in $(2.4),(2.5)$ by more general sequences $\sigma_{j}$ and generalising also the underlying resolutions of unity based on (2.2), (2.3). The most recent and also most advanced paper in this direction is [10], where one finds also atomic decompositions and local means. There seems to be a good chance to extend the Theorem to these spaces.

EXAMPlE 13. All the above (unweighted and weighted) spaces are (inhomogeneous) isotropic spaces. Especially the Russian school dealt from the very beginning of the theory of function spaces with anisotropic generalisations of isotropic Sobolev and Besov spaces. This results nowadays in anisotropic spaces $A_{p q}^{s, a}\left(\mathbb{R}^{n}\right)$ of type $(2.4),(2.5)$ with the anisotropy

$$
a=\left(a_{1}, \ldots, a_{n}\right), \quad a_{1} \geq \cdots \geq a_{n}>0, \quad \sum_{j=1}^{n} a_{j}=n,
$$

and an anisotropic conterpart of (2.2), (2.3). Basic assertions and references may be found in [26], Chapter 4. The recent theory of these spaces is now in many respects at the same level as the theory of the isotropic spaces, including atomic decompositions and representations in terms of local means. We refer to [9] and [5] and the literature mentioned there. Starting from an anisotropic version of an $L_{2}\left(\mathbb{R}^{n}\right)$-wavelet basis (1.1) - (1.4) (if exists) then one has a good chance to extend the above Theorem to anisotropic spaces of this type. In case of related anisotropic Lemarié-Meyer wavelets we refer to [2], [3].

EXAMPLE 14. Let $M$ be a compact or non-compact $C^{\infty}$ Riemannian manifold (with positive injectivity radius and bounded geometry). The theory of the spaces $A_{p q}^{s}(M)$ has been developed in [37], Chapter 7, based on the papers mentioned there. The recent theory may be found in [30], [31], [32] and the references given there, including atomic 
decompositions. If there are $L_{2}(M)$-wavelet bases of the above type on $M$ then it might be possible to extend the Theorem to the spaces $A_{p q}^{s}(M)$.

\section{References}

[1] D. R. Adams and L. I. Hedberg, Function Spaces and Potential Theory, Springer, Berlin, 1996.

[2] M. Z. Berkolaiko and I. Ya. Novikov, Unconditional bases in spaces of functions of anisotropic smoothness, Trudy Mat. Inst. Steklov 204 (1993), 35-51 (in Russian); Engl. translation: Proc. Steklov Inst. Math. 204 (1994), 27-41.

[3] M. Z. Berkolaiko and I. Ya. Novikov, Bases of splashes and linear operators in anisotropic Lizorkin-Triebel spaces, Trudy Mat. Inst. Steklov 210 (1995), 5-30 (in Russian); Engl. translation: Proc. Steklov Inst. Math. 210 (1995), 2-21.

[4] Z. Ciesielski, On the isomorphism of $H_{\alpha}$ and $m$, Bull. Acad. Polon. Sci. Sér. Sci. Math. Astr. Phys. 8 (1960), 217-222.

[5] S. Dachkovski, Anisotropic function spaces and related semi-linear hypoelliptic equations, Math. Nachr. 248-249 (2003), 40-61.

[6] I. Daubechies, Orthonormal bases of compactly supported wavelets, Comm. Pure Appl. Math. 41 (1988), 909-996.

[7] I. Daubechies, Ten Lectures on Wavelets, CBMS-NSF Regional Conf. Series Appl. Math. SIAM, Philadelphia, 1992.

[8] D. E. Edmunds and H. Triebel, Function Spaces, Entropy Numbers, Differential Operators, Cambridge Univ. Press, Cambridge, 1996.

[9] W. Farkas, Atomic and subatomic decompositions in anisotropic function spaces, Math. Nachr. 209 (2000), 83-113.

[10] W. Farkas and H.-G. Leopold, Characterisations of function spaces of generalised smoothness, Annali Math. Pura Appl. (to appear).

[11] M. Frazier and B. Jawerth, Decomposition of Besov spaces, Indiana Univ. Math. Journ. 34 (1985), 777-799.

[12] M. Frazier and B. Jawerth, A discrete transform and decompositions of distribution spaces, Journ. Funct. Anal. 93 (1990), 34-170.

[13] M. Frazier, B. Jawerth and G. Weiss, Littlewood-Paley Theory and the Study of Function Spaces, CBMS Regional Conf. Series Math. AMS, Providence, 1991.

[14] D. Haroske and H. Triebel, Entropy numbers in weighted function spaces and eigenvalue distributions of some degenerate pseudodifferential operators I, Math. Nachr. 167 (1994), 131-156.

[15] D. Haroske and H. Triebel, Entropy numbers in weighted function spaces and eigenvalue distributions of some degenerate pseudodifferential operators II, Math. Nachr. 168 (1994), 109-137.

[16] E. Hernández and G. Weiss, A First Course on Wavelets, CRC Press, Boca Raton, 1996.

[17] G. Kyriazis and P. Petrushev, New bases for Triebel-Lizorkin and Besov spaces, Trans. Amer. Math. Soc. 354 (2001), 749-776.

[18] B. Maurey, Isomorphismes entre espaces $H_{1}$, Acta Math. 145 (1980), 79-120.

[19] Y. Meyer, Wavelets and Operators, Cambridge Univ. Press, Cambridge, 1992.

[20] S. Moura, Function spaces of generalised smoothness, Dissertationes Math. 398 (2001).

[21] J. Peetre, New Thoughts on Besov Spaces, Duke Univ., Durham, 1976. 
[22] S. Roudenko, Matrix-weighted Besov spaces, Trans. AMS 355 (2002), 273-314.

[23] T. Runst and W. Sickel, Sobolev Spaces of Fractional Order, Nemytskij Operators, and Nonlinear Partial Differential Equations, W. de Gruyter, Berlin, 1996.

[24] V. S. Rychkov, On a theorem of Bui, Paluszyński, and Taibleson, Proc. Steklov Inst. Math. 227 (1999), 280-292.

[25] V. S. Rychkov, Littlewood-Paley theory and function spaces with $A_{p}^{\text {loc }}$ weights, Math. Nachr. 224 (2001), 145-180.

[26] H.-J. Schmeisser and H. Triebel, Topics in Fourier Analysis and Function Spaces, Wiley, Chichester, 1987.

[27] T. Schott, Function spaces with exponential weights I, Math. Nachr. 189 (1998), 221-242.

[28] T. Schott, Function spaces with exponential weights II, Math. Nachr. 196 (1998), 231-250.

[29] T. Schott, Pseudodifferential operators in function spaces with exponential weights, Math. Nachr. 200 (1999), 119-149.

[30] L. Skrzypczak, Atomic decompositions on manifolds with bounded geometry, Forum Math. 10 (1998), 19-38.

[31] L. Skrzypczak, Mapping properties of pseudodifferential operators on manifolds with bounded geometry, Journ. London Math. Soc. 57 (1998), 721-738.

[32] L. Skrzypczak, Heat extensions, optimal atomic decompositions and Sobolev embeddings in presence of symmetries on mainfolds, Math. Zeitschr. 243 (2003), 745-773.

[33] R. H. Torres, Boundedness results for operators with singular kernels on distribution spaces, Memoirs Amer. Math. Soc. 442, Amer. Math. Soc., Providence, 1991.

[34] H. Triebel, Über die Existenz von Schauderbasen in Sobolev-Besov-Räumen. Isomorphiebeziehungen, Studia Math. 46 (1973), 83-100.

[35] H. Triebel, Interpolation Theory, Function Spaces, Differential Operators, North-Holland, Amsterdam, 1978.

[36] H. Triebel, Theory of Function Spaces, Birkhäuser, Basel, 1983.

[37] H. Triebel, Theory of Function Spaces, II, Birkhäuser, Basel, 1992.

[38] H. Triebel, Fractals and Spectra, Birkhäuser, Basel, 1997.

[39] H. Triebel, The Structure of Functions, Birkhäuser, Basel, 2001.

[40] H. Triebel, Fractal characteristics of measures; an approach via function spaces, Journ. Fourier Anal. Appl. 9 (2003), 411-431.

[41] H. Triebel, Characterisation of function spaces via mollification; fractal quantities for distributions, Journ. Function Spaces Appl. 1 (2003), 75-89.

[42] P. Wojtaszczyk, A Mathematical Introduction to Wavelets, Cambridge Univ. Press, Cambridge, 1997. 